INPLASY

PROTOCOL

To cite: Lang et al. Pulse electromagnetic field for treating postmenopausal osteoporosis: A systematic review and meta-analysis of randomized controlled trials. Inplasy protocol 2021110066. doi:

10.37766/inplasy2021.11.0066

Received: 17 November 2021

Published: 17 November 2021

Corresponding author:

Ma Xinlong

dashudoctor@163.com

Author Affiliation:

Tianjin Hospital

Support: NSFC.

Review Stage at time of this submission: Preliminary

searches.

Conflicts of interest:

None declared.

\section{Pulse electromagnetic field for treating postmenopausal osteoporosis: A systematic review and meta-analysis of randomized controlled trials}

\author{
Lang, S1; Ma, JX2; Wang, Y3; Gong, SW4; Chen, HT5; Ma, X6.
}

Review question / Objective: The aim of this meta-analysis of randomized controlled trials is to evaluate the efficacy and safety of PEMF in the treatment of postmenopausal osteoporosis.

Information sources: Two authors (SL and SWG) independently searched 4 Chinese databases (China National Knowledge Infrastructure (CNKI), Chinese BioMedical (CBM) Literature Database, Wanfang, and VIP) and 4 English databases (Web of Science, PubMed, Cochrane Library, and Embase) from inception to September 10, 2021 to acquire potentially eligible studies. WHO International Clinical Trials Registry Platform (ICTRP) and Chinese Clinical Trial Registry (ChiCTR) were checked to identify the studies with potential composite inclusion criteria. The detailed search strategy for each database is provided in SupplementaryMaterials.

INPLASY registration number: This protocol was registered with the International Platform of Registered Systematic Review and Meta-Analysis Protocols (INPLASY) on 17 November 2021 and was last updated on 17 November 2021 (registration number INPLASY2021110066).

\section{INTRODUCTION}

Review question / Objective: The aim of this meta-analysis of randomized controlled trials is to evaluate the efficacy and safety of PEMF in the treatment of postmenopausal osteoporosis.
Condition being studied: Postmenopausal osteoporosis, as a type of metabolic bone disease, is a worldwide public health problem with a high incidence and huge economic cost. It is estimated that the number of osteoporosis patients is 200 million people currently and will reach 319 million in 2040. Moreover, it costs about \$ 25.3 billion annually. Osteoporosis can 
significantly increase the fracture risk, especially for elderly women. It is worth noting that some fractures are fatal, such as hip fractures. Therefore, it is crucial to determine the appropriate antiosteoporosis treatment. Anti-osteoporosis pharmacologic therapies are divided into anti-remodeling drugs (including denosumab, raloxifene, bisphosphonates, estrogen, and calcitonin) and bone synthesis drugs (including parathyroid hormone receptor agonists and ramosozumab). However, lifelong pharmacologic therapy generally leads to adverse events, such as breast cancer and deep vein thrombosis, so physical therapy has been appreciated as an alternative therapy with the equivalent efficacy and the lower risk. The American Association of Clinical Endocrinologists recommends physical therapy to reducing bone loss and improving the quality of life of patients in the clinical practice guidelines. Pulsed electromagnetic field (PEMF) has been approved for clinical treatment by the Food and Drug Administration (FDA). PEMF could prevent bone loss and improve symptoms in patients with osteoporosis in randomized controlled studies. Therefore, we first conducted the meta-analysis on the efficacy and safety of PEMF in the treatment of postmenopausal osteoporosis.

\section{METHODS}

Participant or population: All participants with postmenopausal osteoporosis were included regardless of race, age, nationality, course of the menopause, and osteoporosis. The diagnostic criterion for osteoporosis is that the $T$ value of bone mineral density (BMD) $\leq \mathbf{2 . 5}$, as set by the WHO.

Intervention: Experimental interventions included PEMF alone or in combination with conventional medications that recommended by the South American Menopause Society and National Osteoporosis Foundation in the clinical guideline on the treatment and prevention of postmenopausal osteoporosis. Conventional medications for postmenopausal osteoporosis involve calcium, vitamin $D$, alfacalcidol, salmon calcitonin, diphosphonate, and estrogens. There was no restriction on frequency or intensity of PEMF. The comparison was made according to the following relationships: PEMF versus placebo, PEMF versus conventional medications, and PEMF plus conventional medications versus conventional medications.

Comparator: Control interventions included placebo or conventional medications recommended by the South American Menopause Society and National Osteoporosis Foundation in the clinical guideline on the treatment and prevention of postmenopausal osteoporosis.

Study designs to be included: All parallelgroup randomized controlled trials (RCTs) were included in the analysis regardless of publication date.

Eligibility criteria: Firstly, research types including case reports, reviews, animal or cell experiments, and other non-RCT studies were excluded. Secondly, RCTs with unextractable data were excluded. Thirdly, simply published abstracts were excluded. Fourthly, conventional medications which are not recommended by the clinical guideline of South American Menopause Society and National Osteoporosis Foundation were excluded. Fifthly, the patients suffering from other types of osteoporosis, instead of postmenopausal osteoporosis, were excluded.

Information sources: Two authors (SL and SWG) independently searched 4 Chinese databases (China National Knowledge Infrastructure (CNKI), Chinese BioMedical (CBM) Literature Database, Wanfang, and VIP) and 4 English databases (Web of Science, PubMed, Cochrane Library, and Embase) from inception to September 10, 2021 to acquire potentially eligible studies. WHO International Clinical Trials Registry Platform (ICTRP) and Chinese Clinical Trial Registry (ChiCTR) were checked to identify the studies with potential composite inclusion criteria. The detailed search 
strategy for each database is provided in SupplementaryMaterials.

Main outcome(s): Primary outcomes included the BMD of lumbar vertebra, femur and ward's triangle. Secondary outcomes included visual analogue scale (VAS), bio $c$ chemical markers of alkaline phosphatase (ALP), osteocalcin, bonespecific alkaline phosphatase (BSAP), type I collagen carboxy-terminal peptide (CTX), and adverse events. VAS is a scale for evaluating pain. The higher score means the more severe pain.

Quality assessment / Risk of bias analysis: Two reviewers (SL and JXM) independently evaluated eligible studies with the cochrane risk of bias tool from 7 aspects: allocation concealment, random sequence generation, blinding of outcome assessment, blinding of participants and personnel, selective reporting, incomplete outcome data, and other bias. Evaluation results were classified into 3 levels: unclear, low risk, and high risk. If the two reviewers disagreed on the assessment results, the third reviewer (XLM) resolved these differences.Two authors (SL and SWG) independently searched 4 Chinese databases (China National Knowledge Infrastructure (CNKI), Chinese BioMedical (CBM) Literature Database, Wanfang, and VIP) and 4 English databases (Web of Science, PubMed, Cochrane Library, and Embase) from inception to September 10, 2021 to acquire potentially eligible studies. WHO International Clinical Trials Registry Platform (ICTRP) and Chinese Clinical Trial Registry (ChiCTR) were checked to identify the studies with potential composite inclusion criteria. The detailed search strategy for each database is provided in SupplementaryMaterials.

Strategy of data synthesis: Adverse events were analyzed with risk ratio (RR) and $95 \%$ confidence interval $(95 \% \mathrm{Cl})$. Other continuous variables were calculated with mean difference (MD) or standardized mean difference (SMD) with $95 \% \mathrm{Cl}$. In order to estimate the effect size, when the chi-square test $P$ value $>0.1$ or $12<50 \%$, a fixed-effect model was adopted, otherwise the random-effect model was used.

Subgroup analysis: Subgroup analysis through different PEMF frequencies and intensities.

Sensitivity analysis: To explore the source of heterogeneity, meta-regression was performed to detect potential confounding factors. Five regressors were explored: the year of publication, age, follow-up time, sample size, and course of the PEMF. In order to enhance the stability of the results, the sensitivity analysis was performed. Publication bias was detected when there were more than 10 studies in the metaanalysis. In order to enhance the stability of the results, the sensitivity analysis was performed. Publication bias was detected when there were more than 10 studies in the meta-analysis.

\section{Country(ies) involved: China.}

Keywords: Pulse electromagnetic field, Postmenopausal osteoporosis, Systematic review, Meta-analysis.

Contributions of each author:

Author 1 - Lang Shuang.

Author 2 - Ma Jianxiong.

Author 3 - Wang Yan.

Author 4 - Gong Shuwei.

Author 5 - Chen Hengting.

Author 6 - Ma Xinlong. 